九州大学学術情報リポジトリ

Kyushu University Institutional Repository

\title{
A note on odd unimodular Euclidean lattices
}

Nozaki, Hiroshi

Graduate School of Mathematics, Kyushu University: Student (M2) : Algebraic Combinatorics

http://hdl. handle. net/2324/14752

出版情報：Archiv der Mathematik. 86 (3)，pp.226-230，2006-03-16. Birkhäuser Basel バージョン：

権利関係 : 


\title{
A NOTE ON ODD UNIMODULAR EUCLIDEAN LATTICES
}

\author{
HIROSHI NOZAKI
}

\author{
Graduate School of Mathematics Kyushu University \\ Hakozaki 6-10-1 Higashi-ku, Fukuoka, 812-8581 Japan \\ ma204018@math.kyushu-u.ac.jp
}

\begin{abstract}
We prove that the theta series of any odd unimodular Euclidean Lattice is not congruent to 1 modulo any odd prime $p$.
\end{abstract}

\section{INTRODUCTION}

It is known that there are many even unimodular lattices in $R^{n}$ whose theta series are congruent to 1 modulo prime p. See e.g., Bayer-Fluckiger [1], Dummigan and Tiep [3] and the papers referred there. The following problem was recently asked by Yuichiro Taguchi.

Problem 1.1. Is there any unimodular lattice $\Lambda \subset R^{n}$ with $n$ odd whose theta series $\Theta_{\Lambda}$ satisfy

$$
\Theta_{\Lambda} \equiv 1 \quad(\bmod p)
$$

for an odd prime $p$ ?

In the meantime, Taguchi noticed himself that it is an implicit consequence of the paper of Koblitz [4, pages 202-203] that there exists no such lattice. However, it seems that the proof is difficult since it heavily depends on the deep results of Katz and Kohnen. The purpose of this paper is to give an elementary and simple proof of this solution. Also, we prove it in a slightly general form as follows.

Theorem 1.1. For any odd unimodular Euclidean lattice and for any odd prime $p$, its theta series cannot be congruent to 1 modulo $p$.

\section{PRELIMINARIES}

We have several definitions as follows.

The dual of a lattice $\Lambda \subset R^{n}$ is the lattice

$$
\Lambda^{\#}:=\left\{y \in R^{n} \mid\langle y \mid x\rangle \in \mathbb{Z}, \forall x \in \Lambda\right\} .
$$

The lattice $\Lambda$ is called integral if $\Lambda \subset \Lambda^{\#}$. An integral lattice $\Lambda$ is called even if $\langle x \mid x\rangle \in 2 \mathbb{Z}$ for all $x \in \Lambda$. It is called odd otherwise. An integral lattice is called selfdual (or unimodular) if $\Lambda^{\#}=\Lambda$. The shadow $S(\Lambda)$ of a selfdual lattice $\Lambda$ is

$$
S(\Lambda)= \begin{cases}\Lambda, & \text { if } \Lambda \text { is even } \\ \Lambda_{0}^{\#} \backslash \Lambda, & \text { if } \Lambda \text { is odd }\end{cases}
$$

where $\Lambda_{0}=\{x \in \Lambda \mid\langle x \mid x\rangle \equiv 0 \bmod 2\}$.

Mathematical Subject Classification(2000): Primary 11F33 Secondary 11H06, 52C07 
We write $q:=e^{\pi i z}$.

$$
\begin{gathered}
\theta_{3}(z):=\sum_{m \in \mathbb{Z}} q^{m^{2}}=1+2 q+2 q^{4} \cdots \\
\theta_{2}(z):=\sum_{m \in \mathbb{Z}+\frac{1}{2}} q^{m^{2}}=2 q^{\frac{1}{4}}\left(1+q^{2}+\cdots\right) \\
\theta_{4}(z):=\sum_{m \in \mathbb{Z}}(-q)^{m^{2}}=1-2 q+2 q^{4}-\cdots
\end{gathered}
$$

The theta series of $\Lambda$ is

$$
\Theta_{\Lambda}(z):=\sum_{x \in \Lambda} q^{\langle x \mid x\rangle}
$$

We collect the results we need.

Theorem 2.1. (A special case of Dummigan-Tiep [3, Theorem 3.2]) Let $p$ be an odd prime and $\varphi$ denote the Euler function. Define $M=l \varphi(p)$ as follows :

(1) If $p \equiv 1(\bmod 4)$, then $M=2 \varphi(p)$.

(2) If $p \equiv 3(\bmod 4)$, then $M=4 \varphi(p)$.

Then there is an even unimodular lattice $\Lambda$ of rank $M$ such that $\Theta_{\Lambda} \equiv 1(\bmod p)$.

Theorem 2.2. (Originally due to Hecke, See Conway-Sloane [2, pages 187-188]) If $\Lambda$ is a unimodular lattice with rank $n$ then

$$
\Theta_{\Lambda}=\theta_{3}^{n}+c_{1} \theta_{3}^{n-8} \Delta_{8}+\cdots+c_{[n / 8]} \theta_{3}^{n-8[n / 8]} \Delta_{8}^{[n / 8]}
$$

where the $c_{i}$ are integers and $\Delta_{8}(z)$ is the cusp form

$$
\Delta_{8}(z)=\frac{1}{16} \theta_{2}(z)^{4} \theta_{4}(z)^{4}=q \prod_{m=1}^{\infty}\left\{\left(1-q^{2 m-1}\right)\left(1-q^{4 m}\right)\right\}^{8} .
$$

Theorem 2.3. (Pache [5, Proposition 13]) In the notation of Theorem 2.2,

$$
\Theta_{\Lambda}=\theta_{3}^{n}+c_{1} \theta_{3}^{n-8} \Delta_{8}+\cdots+c_{k} \theta_{3}^{n-8 k} \Delta_{8}^{k}
$$

where $k=\frac{1}{8}(n-\sigma(\Lambda))(\sigma(\Lambda):=4 \min \{\langle x \mid x\rangle \mid x \in S(\Lambda)\}$ where $S(\Lambda)$ means the shadow of the lattice $\Lambda)$. Moreover, we have $c_{k}=(-1)^{k} 2^{-n+12 k}\left|S(\Lambda)_{(n-8 k) / 4}\right|$, where $S(\Lambda)_{m}:=\{x \in S(\Lambda) \mid\langle x \mid x\rangle=m\}$.

Since $\varphi(p)=p-1$,

$$
M= \begin{cases}8 t, & \text { if } p=4 t+1, t \in \mathbb{N} \\ 8(2 t-1), & \text { if } p=4 t-1, t \in \mathbb{N}\end{cases}
$$

For every odd prime $p$, we can pick out an even unimodular lattice $\Lambda$ of rank $M$ such that $\Theta_{\Lambda} \equiv 1(\bmod p)($ by Theorem 2.1$)$. Since $\Lambda$ is a unimodular lattice, there exist $c_{i} \in \mathbb{Z}$ such that

$$
\Theta_{\Lambda}=\theta_{3}^{M}+c_{1} \theta_{3}^{M-8} \Delta_{8}+\cdots+c_{k} \theta_{3}^{M-8 k} \Delta_{8}^{k}
$$

where $\sigma(\Lambda)=M-8 k$ and $c_{k}=(-1)^{k} 2^{-M+12 k}\left|S(\Lambda)_{(M-8 k) / 4}\right|$ (by Theorem 2.3).

Moreover $\Lambda$ is even. Hence $S(\Lambda)=\Lambda$ and $\sigma(\Lambda)=0$. Therefore

$$
c_{k}=(-1)^{k} 2^{-M+12 k}\left|\Lambda_{0}\right|=(-1)^{k} 2^{-M+12 k} .
$$

(1)If $p=4 t+1$, then $M=8 t$. Since $\sigma(\Lambda)=M-8 k=0, k=t$. Therefore 


$$
c_{k}=(-1)^{t} 2^{4 t}=(-1)^{t} 2^{p-1} \equiv(-1)^{t}(\bmod p) .
$$

(2)If $p=4 t-1$, then $M=8(2 t-1)$. Since $\sigma(\Lambda)=M-8 k=0, k=2 t-1$. Therefore

$$
c_{k}=(-1)^{2 t-1} 2^{8 t-4}=-2^{2(p-1)} \equiv-1(\bmod p) .
$$

The facts mentioned above prove the following proposition.

Proposition 2.1. Let $p$ be an odd prime. Define $M=4 \varphi(p)$. There is an even unimodular lattice $\Lambda$ of rank $M$ such that

$$
\Theta_{\Lambda}=\theta_{3}^{M}+c_{1} \theta_{3}^{M-8} \Delta_{8}+\cdots+c_{M / 8} \Delta_{8}^{M / 8} \equiv 1 \quad(\bmod p)
$$

where $c_{i} \in \mathbb{Z}, c_{M / 8} \not \equiv 0(\bmod p)$ and $M / 8 \in \mathbb{N}$.

If $p=4 n+1$, the rank of $\Lambda$ is $2 \varphi(p)$ in Theorem 2.1. Then $\Theta_{\Lambda \oplus \Lambda}=\Theta_{\Lambda}{ }^{2} \equiv 1$ $(\bmod p)$ and $\Lambda \oplus \Lambda$ is an even unimodular lattice and $\operatorname{rank} 4 \varphi(p)$.

\section{Proof of Theorem 1.1}

In order to prove Theorem 1.1, we prove the following two results. Theorem 3.1 is independent of the existence of the lattice.

Theorem 3.1. Let $n$ be any integer which is not divisible by 8. For any odd prime $p$, there does not exist $\left\{a_{0}, a_{1}, \cdots, a_{[n / 8]}\right\}$ such that

$$
\sum_{i=0}^{[n / 8]} a_{i} \theta_{3}^{n-8 i} \Delta_{8}^{i} \equiv 1 \quad(\bmod p)
$$

where $a_{0}=1$ and $a_{i}$ are integers.

Proof. Assume the contrary. Then there exists $\left\{a_{1}, \cdots, a_{[n / 8]}\right\}$ such that

$$
\Theta_{\Lambda}=\theta_{3}^{n}+a_{1} \theta_{3}^{n-8} \Delta_{8}+\cdots+a_{[n / 8]} \theta_{3}^{n-8[n / 8]} \Delta_{8}^{[n / 8]} \equiv 1 \quad(\bmod p) .
$$

Since $n$ is not divisible by $8, n-8[n / 8] \neq 0$.

By Proposition 2.1 there exists an even unimodular lattice $\Lambda^{\prime}$ with rank $M$ such that

$$
\Theta_{\Lambda^{\prime}}=\theta_{3}^{M}+c_{1} \theta_{3}^{M-8} \Delta_{8}+\cdots+c_{M / 8} \Delta_{8}^{M / 8} \equiv 1 \quad(\bmod p)
$$

where $c_{i} \in \mathbb{Z}$ and $c_{M / 8} \not \equiv 0(\bmod p)$.

$$
\Theta_{\Lambda^{\prime}}{ }^{n}-\Theta_{\Lambda}{ }^{M}=b_{1} \theta_{3}^{M n-8} \Delta_{8}+b_{2} \theta_{3}^{M n-16} \Delta_{8}^{2}+\cdots+b_{M n / 8} \Delta_{8}^{M n / 8} \equiv 0 \quad(\bmod p)
$$

where $b_{i} \in \mathbb{Z}$. Since $\theta_{3}^{M n-8 i} \Delta_{8}^{i}=q^{i}(1+\cdots)$, then $b_{1} \equiv 0, b_{2} \equiv 0, \cdots$ and $b_{M n / 8} \equiv 0$ $(\bmod p)$. However this contradicts $b_{M n / 8}=\left(c_{M / 8}\right)^{n} \not \equiv 0(\bmod p)$.

Theorem 3.2. Let $n$ be any integer which is divisible by 8. For any odd unimodular lattice $\Lambda$ of rank $n$ and for any odd prime $p$,

$$
\Theta_{\Lambda}=\sum_{i=0}^{k} a_{i} \theta_{3}^{n-8 i} \Delta_{8}^{i} \not \equiv 1 \quad(\bmod p)
$$

where $a_{0}=1, a_{i}$ are integers and $k=\frac{1}{8}(n-\sigma(\Lambda))$.

Proof. We have $\sigma(\Lambda)=0$ if and only if $\Lambda$ is even. Therefore $\sigma(\Lambda)=n-8 k \neq 0$. Remains of proof is similar to proof of theorem 3.1. 
Acknowledgements. The author thanks Eiichi Bannai for suggesting this research problem and providing useful informations. The author also thanks Boris Venkov for his suggestion (communicated through Eiichi Bannai) that the problem might be solved purely at the level of the theta series.

\section{REFERENCES}

[1] E. Bayer-Fluckiger: "Definite unimodular lattices having an automorphism of given characteristic polynomial ", Comment. Math. Helvetici 59 (1984), 509-538

[2] J.H. Conway, N.J.A. Sloane: "Sphere Packings, Lattices and Groups", third edition, (1999).

[3] N. Dummigan and P.H. Tiep: "Congruences for Certain Theta Series", Journal of Number Theory 71,86-105(1998).

[4] N. Koblitz: " $p$-Adic Congruences and Modular Forms of Half Integer Weight", Math. Annalen 274 (1986), 199-220.

[5] Claude Pache: "Shells of selfdual lattices viewed as spherical designs", (2004), preprint. 